\title{
FOCUSED SIMULATED ANNEALING SEARCH: AN APPLICATION TO JOB-SHOP SCHEDULING
}

\author{
Norman M. Sadeh \\ The Robotics Institute \\ Carnegie Mellon University \\ Pittsburgh, PA 15213, U.S.A. \\ sadeh@cs.cmu.edu
}

\author{
Yoichiro Nakakuki \\ NEC C\&C Research Laboratories \\ 4-1-1 Miyazaki, Miyamae-ku \\ Kawasaki 216, JAPAN \\ nakakuki@swl.cl.nec.co.jp
}

\begin{abstract}
This paper presents a simulated annealing search procedure developed to solve job shop scheduling problems simultaneously subject to tardiness and inventory costs. The procedure is shown to significantly increase schedule quality compared to multiple combinations of dispatch rules and release policies, though at the expense of intense computational efforts. A meta-heuristic procedure is developed that aims at increasing the efficiency of simulated annealing by dynamically inflating the costs associated with major inefficiencies in the current solution. Three different variations of this procedure are considered. One of these variations is shown to yield significant reductions in computation time, especially on problems where search is more likely to get trapped in local minima. We analyze why this variation of the meta-heuristic is more effective than the others.
\end{abstract}




\section{Introduction}

Over the past several years, with the advent of ever more powerful computers, stochastic procedures such as Simulated Annealing (SA) [14, 2] (and improved variations exploiting Tabu Search principles [9, 10]) or Genetic Algorithms (GAs) [11] have attracted the attention of a growing number of researchers. This interest has been fueled by both experimental and theoretical results indicating that, if properly designed and if given enough time, these procedures are often capable of finding near-optimal solutions to complex optimization problems.

This paper presents results obtained using SA to find solutions to job shop scheduling problems where the objective is to minimize the sum of weighted tardiness and inventory costs (both work-in-process inventory and finished-goods inventory costs). The model is particularly attractive as it is compatible with the Just-In-Time objective of meeting customer demand in a timely yet cost-effective manner. In the scheduling literature, this objective function is known to be irregular, as its value may sometimes be decreased by delaying the execution of some operations [3]. As will be shown, this property needs to be taken into account in the design of SA procedures for this class of problems.

By reference to simpler problems (e.g. the one machine version of this problem), this problem can easily be shown to be NP-hard [5, 6, 22, 23]. Surprisingly enough, despite the "attractiveness" of its modeling assumptions, this problem has been given very little attention in the literature. Two notable exceptions are the work of Tom Morton on resource-pricing heuristics in the context of the Sched-Star system [17] and our earlier work on micro-opportunistic bottleneck-centered techniques in the context of the Micro-Boss factory scheduling system [24].

The first part of this paper presents a SA procedure developed to solve job shop scheduling problems subject to both tardiness and inventory costs. Experimental results are presented comparing the performance of our procedure with that of several other scheduling heuristics. The results corroborate earlier studies performed on other combinatorial optimization problems. They indicate that SA consistently produces high quality solutions, often significantly outperforming other scheduling heuristics, though at the expense of intensive computational efforts. In the second part of this pa-

per, we introduce "Focused Simulated Annealing" (FSA), a meta-heuristic procedure that aims at improving the efficiency of SA search. The idea behind FSA is that by 
dynamically inflating the costs associated with major inefficiencies in the existing solution, it is possible to focus the procedure and force it to get rid of these inefficiencies. By iteratively inflating costs in different subproblems, FSA can reduce the chances that the procedure gets trapped in local minima. Three variations of this meta-heuristic are considered that differ in the type of subproblems they rely on: job subproblems, resource subproblems, or operation subproblems. Experimental results comparing these three variations of the meta-heuristic against the original SA procedure show that the job-based meta-heuristic significantly improves performance, especially on problems where search is particularly likely to get caught in local minima. We further analyze why this variation of the meta-heuristic is more effective than the others, trying to shed some light on why, in general. some decompositions are likely to work better than others for a given SA procedure.

The balance of this paper is organized as follows. Section 2 provides a formal definition of the job shop scheduling problem considered in this study. Section 3 presents a SA search procedure developed for this problem. Section 4 reports experimental results comparing the performance of the procedure against that of other scheduling heuristics. The concept of Focused Simulated Annealing is introduced in Section 5 and three variations of this meta-heuristic procedure are developed for the job shop scheduling problem with tardiness and inventory costs. Performance of these meta-heuristics is reported in Section 6. These results are further discussed and analyzed in Section 7. Section 8 presents some concluding remarks.

\section{The Job Shop Scheduling Problem with Tardiness and In- ventory Costs}

We consider a factory, in which a finite set of jobs, $J=\left\{j_{1}, j_{2}, \cdots, j_{n}\right\}$, has to be scheduled on a finite set of resources, $R E S=\left\{R_{1}, R_{2}, \cdots, R_{m}\right\}$. The jobs are assumed to be known ahead of time and all resources are assumed to be available over the entire scheduling horizon. Each job $j_{l}$ requires performing a set of manufacturing operations $O^{l}=\left\{O_{1}^{l}, O_{2}^{l}, \cdots O_{n_{l}}^{l}\right\}$ and, ideally, should be completed by a specific due date, $d d_{l}$, for delivery to a customer. Precedence constraints specify a complete order in which operations in each job have to be performed. By convention, we assume that operation $O_{i}^{l}$ has to be completed before operation $O_{i+1}^{l}$ can start $\left(i=1,2, \cdots, n_{l}-1\right)$.

Each job $j_{l}$ has an earliest acceptable release date, $\operatorname{erd} d_{l}$, before which it cannot start, 
e.g. because the raw materials or components required by this job cannot be delivered before that date. Each job also has a latest acceptable completion date (or deadline), $l c d_{l}$, by which it should absolutely be completed, e.g. because the customer would otherwise refuse delivery of the order. For each job, we assume that $e r d_{l} \leq d d_{l} \leq l c d_{l}$. Furthermore, we assume that these constraints are loose enough to always allow for the construction of a feasible schedule (i.e. we are not concerned with the detection of infeasible problems).

This paper considers problems in which each operation $O_{i}^{l}$ requires a single resource $R_{i}^{l} \in R E S$ and the order in which a job visits different resources varies from one job to another. Each resource can only process one operation at a time and is nonpreemptable. The duration $d u_{i}^{l}$ of each operation $O_{i}^{l}$ is assumed to be known.

The problem requires finding a schedule (i.e. a set of start times, st $t_{i}^{l}$, for all operations, $O_{i}^{l}$ ) that satisfies all these constraints while minimizing the sum of tardiness costs and inventory costs of all the jobs.

Specifically, each job $j_{l}$ incurs a positive marginal tardiness cost $\operatorname{tar} d_{l}$ for each unit of time that it finishes past its due date $d d_{l}$. Marginal tardiness costs generally correspond to tardiness penalties, interests on lost profits, loss of customer goodwill, etc. The total tardiness cost of job $j_{l}$, in a given schedule, is measured as: $T A R D^{l}=$ $\operatorname{tard}_{l} \cdot \operatorname{MAX}\left(0, C_{l}-d d_{l}\right)$ where $C_{l}$ is the completion date of job $j_{l}$. That is $C_{l}=$ $s t_{n_{l}}^{l}+d u_{n_{l}}^{l}$, where $O_{n_{l}}^{l}$ is the last operation of $j_{l}$.

Inventory costs on the other hand can be introduced at the level of any operation in a job. In our model, each operation $O_{i}^{l}$ can have its own non-negative marginal inventory cost, $i n_{i}^{l}$. This is the marginal cost that is incurred for each unit of time that spans between the start time of this operation and either the completion date of the job or its due date, whichever is larger. In other words, the total inventory cost introduced by an operation $O_{i}^{l}$ in a given schedule is:

$$
I N V_{i}^{l}=i n_{i}^{l} \cdot\left(M A X\left(C_{l}, d d_{l}\right)-s t_{i}^{l}\right)
$$

Typically, the first operation in a job introduces marginal inventory costs that correspond to interests on the costs of raw materials, interests on processing costs (for that first operation), and marginal holding costs. Following operations introduce additional inventory costs such as interests on processing costs, interests on the costs of additional raw materials or components required by these operations, etc. Additional details on this model can be found in [23, 24]. 
The total cost of a schedule is:

$$
\sum_{l \in J} T A R D^{l}+\sum_{l \in J} \sum_{i=1}^{n_{l}} I N V_{i}^{l}
$$

For reasons that will become clearer in Section 5, it is often useful to look at the total tardiness and inventory costs of a job as sums of tardiness and inventory costs introduced by each of the operations in the job. For each operation $O_{i}^{l}$, we can define a best start time (or "just-in-time" start time), $b_{s} t_{i}^{l}$, where:

$$
b s t_{i}^{l}= \begin{cases}d d_{l}-d u_{i}^{l} & \left(i=n_{l}\right) \\ b s t_{i+1}-d u_{i}^{l} & \left(1 \leq i<n_{l}\right)\end{cases}
$$

Accordingly, the tardiness cost $T A R D^{l}$ of job $j_{l}$ in a given schedule, can be rewritten as:

$$
T A R D^{l}=\sum_{i=1}^{n_{l}} t \cos t_{i}^{l}
$$

where,

$$
\operatorname{tcost}_{i}^{l}= \begin{cases}\operatorname{tard}_{l} \cdot M A X\left(0, s t_{i}^{l}-b s t_{i}^{l}\right) & (i=1) \\ \operatorname{tard}_{l} \cdot\left\{M A X\left(0, s t_{i}^{l}-b s t_{i}^{l}\right)-M A X\left(0, s t_{i-1}^{l}-b s t_{i-1}^{l}\right)\right\} & \left(1<i<n_{l}\right) \\ \operatorname{tard}_{l} \cdot\left\{M A X\left(0, C_{l}-d d_{l}\right)-M A X\left(0, s t_{i-1}^{l}-b s t_{i-1}^{l}\right)\right\} & \left(i=n_{l}\right)\end{cases}
$$

tcost $t_{i}^{l}$ can be seen as the contribution of operation $O_{i}^{l}$ to the total tardiness cost of job $j_{l}$. Similarly, the total inventory cost of a job $j_{l}$ can be rewritten as:

$$
I N V^{l}=\sum_{i=1}^{n_{l}} i \cos t_{i}^{l}
$$

where:

$$
I N V_{i}^{l}=i \cos t_{i}^{l}= \begin{cases}i n v_{i}^{l} \cdot\left(M A X\left(s t_{i}^{l}+d u_{i}^{l}, d d_{l}\right)-s t_{i}^{l}\right) & \left(i=n_{l}\right) \\ i n v_{i}^{l} \cdot\left(s t_{i+1}^{l}-s t_{i}^{l}\right) & \left(1 \leq i<n_{l}\right)\end{cases}
$$

and $i n v_{i}^{l}=\sum_{k=1}^{i} i n_{k}^{l}$. Accordingly, the total cost of a schedule can also be expressed as:

$$
\sum_{l \in J} T A R D^{l}+\sum_{l \in J} I N V^{l}=\sum_{l \in J} \sum_{i=1}^{n_{l}}\left(t \cos t_{i}^{l}+i \cos t_{i}^{l}\right)
$$

For the sake of simplicity, the remainder of this paper further assumes that time is discrete, i.e. that job due dates/earliest acceptable release dates/latest acceptable completion dates and operation durations can only can only take integer values.

The following section introduces a SA procedure developed for this problem. 


\section{A Simulated Annealing Procedure}

Simulated Annealing (SA) is a general-purpose search procedure that generalizes iterative improvement approaches to combinatorial optimization by sometimes accepting transitions to lower quality solutions so as to avoid getting trapped in local minima $[14,2]$. SA procedures have been successfully applied to a variety of combinatorial optimization problems, including Traveling Salesman Problems [2], Graph Partitioning Problems [12], Graph Coloring Problems [13], Vehicle Routing Problems [21], Design of Integrated Circuits, Minimum Makespan Flow-Shop Scheduling Problems [20], Minimum Makespan Job Shop Scheduling Problems [16, 26], etc.

$$
\begin{aligned}
& T=T_{0} \\
& x=x_{0}(\in S) \text {; } \\
& \text { BestSol }=x_{0} ; \quad M=\operatorname{cost}(\text { BestSol }) \text {; } \\
& \text { while }\left(T>T_{1}\right) \quad\{ \\
& \text { for } i=1, N \text { \{ } \\
& x^{\prime}=\text { neighbor }(x) \text {; } \\
& \text { if }\left(\operatorname{cost}\left(x^{\prime}\right)<\operatorname{cost}(x)\right) \quad\{ \\
& x=x^{\prime} \text {; } \\
& \text { \} } \\
& \text { if }\left(\operatorname{cost}\left(x^{\prime}\right)<M\right) \quad\left\{\text { BestSol }=x^{\prime} ; M=\operatorname{cost}(\text { BestSol }) ;\right\} \\
& \text { \} } \\
& \text { else if }\left(\operatorname{rand}()<\exp \left\{\left(\operatorname{cost}(x)-\operatorname{cost}\left(x^{\prime}\right)\right) / T\right\}\right) \quad x=x^{\prime} \text {; } \\
& \text { if ( } M \text { was not modified in the above loop) } T=T * \alpha \text {; }
\end{aligned}
$$

Fig. 1 Pseudo-code for a Basic SA Search Procedure.

Figure 1 outlines the main steps of a SA search procedure designed to find a solution $x \in S$ that minimizes a real-valued cost function, $\operatorname{cost}(x)$. The procedure starts from an initial solution $x_{0}$ and iteratively moves to other neighboring solutions, while remembering the best solution found so far (BestSol). Typically, the procedure only moves to neighboring solutions that are better than the current one. However, the probability of moving from a solution $x$ to an inferior solution $x^{\prime}$ is greater than zero, thereby allowing the procedure to escape from local minima. rand() is a function that randomly draws a number from a uniform distribution on the interval [0,1]. The 
so-called temperature, $\mathrm{T}$, of the procedure is a parameter controlling the probability of accepting a transition to a lower quality solution. It is initially set to a high value, $T_{0}$, thereby frequently allowing such transitions. If, after $N$ iterations, the best solution found by the procedure has not improved, the temperature parameter $T$ is decremented by a factor $\alpha(0 \leq \alpha \leq 1)$. When the temperature drops below a preset level, $T_{1}$, the procedure stops and the best solution it found (BestSol) is returned (not shown in the pseudo-code in Figure 1).

As indicated earlier, procedures similar to the one outlined above have been successfully applied to other scheduling problems such as the minimum makespan job-shop scheduling problem ${ }^{1}$. When dealing with regular scheduling objectives such as minimum makespan, it is possible to limit search to permutations of operations on the same machine. For instance, in their SA procedure, Van Laarhoven et al. exploit this observation and restrict the neighborhood structure to permutations of consecutive operations on a same machine [26]. In the case of scheduling problems with irregular objectives, such a neighborhood structure would not be sufficient, as it does not allow for the insertion of idle-time in the schedule, which sometimes improves the quality of a solution ${ }^{2}$. Here, two main approaches can be considered. A first approach would be to combine a SA procedure relying on permutation-based neighborhoods with a procedure that inserts idle-time optimally. As it turns out, the problem of inserting idle time optimally in a schedule, given completely specified sequences of operations on each machine, can be formulated as a Linear Programming (LP) problem and, hence, can be solved in polynomial time (See Appendix A for details). When considering the permutation of two operations, the SA procedure would first invoke an idle-time insertion procedure to compute the cost of the best schedule compatible with the new set of sequencing decisions. Based on this cost and the cost of the current solution, the search procedure would probabilistically determine whether or not to accept the transition. Nevertheless, at the present time, the idle time insertion procedures that the authors are aware of for the job shop scheduling problem remain too slow and

\footnotetext{
${ }^{1}$ The makespan of a schedule is the length of the time interval that spans between the start time of the first released job and the end time of the last completed job.

${ }^{2}$ Scheduling problems with regular objective functions have been shown to be reducible to sequencing problems [1]. Given fixed operation sequences on each machine, the schedule obtained by starting each operation as early as possible is undominated. With irregular objectives, this is no longer the case and it is sometimes better to delay the start of some operations. Here, we generically refer to the problem of deciding by how much to delay operations as the problem of "inserting idle time" in the schedule.
} 
would significantly limit the number of solutions that SA could explore in a reasonable amount of time ${ }^{3}$.

Instead, an alternative neighborhood structure was adopted that directly allows for idle time insertion. This structure, which is described below, lends itself to quick updates of the cost function. A possibly more subjective advantage has to do with the fact that the resulting procedure relies solely on SA and hence is not affected by the performance of a separate idle time insertion procedure. Specifically, the neighborhood function used in our implementation randomly selects among three types of modification operators, respectively referred to below as "RIGHT-SHIFT", "LEFT-SHIFT" and "EXCHANGE":

RIGHT-SHIFT This operator randomly chooses a "right-shiftable" operation and increases its start time by one time unit (Figure 2-(a)). An operation is assumed to be "right-shiftable", if it can be shifted by one time unit without bumping into another operation on the same resource or violating the latest acceptable completion date of the job to which it belongs (Figure 2-(b)). Precedence constraints within a job are ignored when determining whether or not an operation can be right-shifted. Instead, as will be seen later, these constraint violations are taken care of by inserting artificial costs in the objective function.

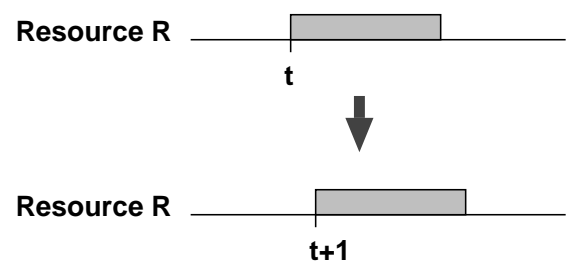

(a) SHIFT-RIGHT

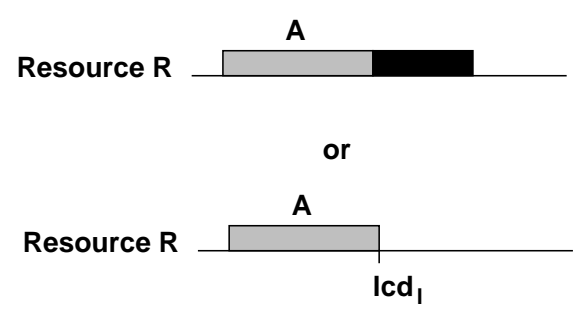

(b) not shiftable

\section{Fig. 2 RIGHT-SHIFT operator}

\footnotetext{
${ }^{3}$ For instance, using the CPLEX Linear Programming package on a DECstation 5000/200, inserting idle time optimally in a 100 operation job shop schedule takes about $1 \mathrm{CPU}$ second. Taking into account similarities between the current schedule and the schedule obtained after permuting the order of two operations on the same resource, it is generally possible to reduce the time required to reoptimize the schedule to about 0.1 to $0.2 \mathrm{CPU}$ seconds. Even under these conditions, a SA run of about 10 minutes would only be able to explore a few thousand solutions.
} 
LEFT-SHIFT This operator is the mirror image of RIGHT-SHIFT. It randomly picks a "left-shiftable" operation and decreases its start time by one time unit (Figure 3-(a)). It is assumed that an operation cannot be shifted left, if it would either bump into an adjacent operation on the same resource (top case in Figure 3 -(b)) or violate the earliest acceptable release date of the job to which it belongs (bottom case in Figure $3-(b))$.

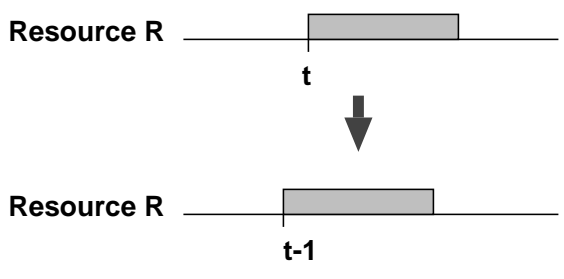

(a) SHIFT-LEFT

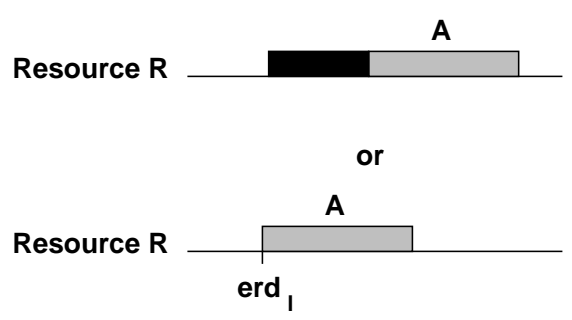

(b) not shiftable

\section{Fig. 3 LEFT-SHIFT operator}

EXCHANGE This operator selects a pair of consecutive operations on a resource and exchanges the order in which the operations are scheduled to be processed on that resource. Specifically, given two consecutive operations, $A$ and $B$ on a resource $\mathrm{R}$, with $A$ preceding $B$ in the current solution, the exchange operator sets the new start time of $B$ to the old start time of $A$ and the new end time of $A$ to the old end time of $B$, as depicted in Figure 4.

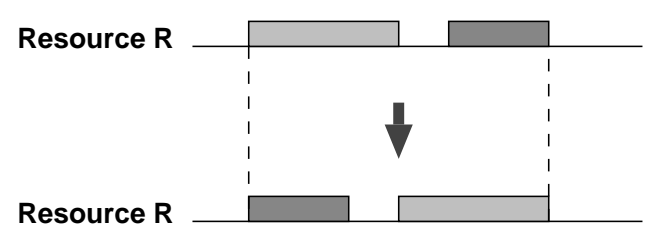

Fig. 4 EXCHANGE operator

In the experiments presented in this paper, the probability of picking the EXCHANGE operator was empirically set to $3 / 7$ while the probabilities of picking a RIGHT- or LEFT-SHIFT operator were both set to $2 / 7$. The initial solution $x_{0}$ used by the SA procedure is randomly generated in such a way that no two operations use 
the same resource at the same time. As with the RIGHT- and LEFT-SHIFT operator, precedence constraints between consecutive operations within a same job are not enforced in the process. Instead these constraints are enforced using artificial costs. If an operation $O_{i}^{l}$ overlaps with a preceding operation $O_{i-1}^{l}$ (within the same job $j_{l}$ ), an artificial cost $f \operatorname{cost}_{i}^{l}$ is introduced in the objective function:

$$
\operatorname{cost}(x)=\sum_{l \in J} \sum_{i=1}^{n_{l}}\left(f \cos t_{i}^{l}+t \cos t_{i}^{l}+i \cos t_{i}^{l}\right)
$$

where $f_{\text {cost }}$ is proportional to the amount of overlap between $O_{i}^{l}$ and its predecessor $O_{i-1}^{l}$. Specifically:

$$
\begin{aligned}
& f \operatorname{cost}_{1}^{l}=0 \\
& f \operatorname{cost}_{i}^{l}=\beta \cdot \max \left(0, s t_{i-1}^{l}+d u_{i-1}^{l}-s t_{i}^{l}\right) \quad(i \geq 2)
\end{aligned}
$$

where $\beta$ is a large positive constant.

The next section summarizes the results of experiments comparing this basic SA procedure with several other scheduling heuristics.

\section{A First Set of Empirical Results}

Performance of this first SA procedure was assessed through comparative studies against a number of other scheduling heuristics. This section summarizes the results of experiments comparing the SA procedure against 39 combinations of well-regarded dispatch rules and release policies (including those combinations that were reported to perform best in the evaluation of the Sched-Star scheduling system [17]) both with and without idle-time optimization, using the LP formulation provided in Appendix A.

Specifically, two types of dispatch rules were considered:

1. A set of five priority dispatch rules that have been reported to be particularly good at reducing tardiness under various scheduling conditions [27]: the Weighted Shortest Processing Time (WSPT) rule, the Earliest Due Date (EDD) rule, the Slack per Remaining Processing Time (SRPT) rule, and two parametric rules, the Weighted Cost OVER Time (WCOVERT) rule and the Apparent Tardiness Cost (ATC) rule (also referred to sometimes as the Rachamadugu\&Morton rule [18]). 
2. An exponential version of the parametric early/tardy dispatch rule recently developed by $\mathrm{Ow}$ and Morton $[22,17]$ and referred to below as EXP-ET. This rule differs from the other 5 in that it can explicitly account for both tardiness and inventory costs.

EXP-ET was successively run in combination with two release policies: an intrinsic release policy that only releases jobs when their priorities become positive, as suggested in [17], and an immediate release policy (IM-REL) that allowed each job to be relased immediately. The other five dispatch rules were also successively run in combination with two release policies: an immediate release policy and the Average Queue Time release policy (AQT) described in [17]. AQT is a parametric release policy that estimates queuing time as a multiple of the average job duration (the look-ahead parameter serving as the multiple). A job's release date is determined by offsetting the due date of the job by the sum of its total duration and its estimated queuing time. In their evaluation of the SCHED-STAR scheduling system, Morton et al. report that the combination of WCOVERT and AQT performed best after their SCHED-STAR system and was within $0.1 \%$ of the best schedule in $42 \%$ of the problems they studied and within $4 \%$ in $70 \%$ of their problems [17]. They also report that the next best scheduling heuristic is EXP-ET in combination with its intrinsic release policy.

Combinations of release policies and dispatch rules with a look-ahead parameter were successively run with four different parameter values that had been identified as producing the best results. By combining these different dispatch rules, release policies and parameter settings a total of 39 heuristics ${ }^{4}$ was obtained.

These 39 combinations of priority dispatch rules and release policies were run in two different ways:

1. On each problem, the best of the 39 schedules produced by these combinations was recorded. In this case, out of the 39 combinations, 13 performed best on at least one of the 40 problems considered in the study. These 13 combinations included 5 of the 6 dispatch rules (SRPT was never best on this set of problems) and all 3 release policies.

\footnotetext{
${ }^{4}$ The 39 combinations were as follows: EXP-ET and its intrinsic policy (times four parameter settings), EXP-ET/IM-REL (times four parameter settings), EDD/AQT (times four parameter settings), EDD/IM-REL, WSPT/AQT (times four parameter settings), WSPT/IM-REL, SRPT/AQT (times four parameter settings), SRPT/IM-REL, WCOVERT/IM-REL (times four parameter settings), WCOVERT/AQT (times four parameter settings), ATC/IM-REL (times four parameter settings), ATC/AQT (times four parameter settings).
} 
2. On each problem, each of the 39 schedules obtained by these combinations was post-processed using an LP program to insert idle time optimally. Again, on each problem, the best of the 39 post-processed schedules was recorded for comparison against the SA procedure. In this case, out of the 39 combinations, 11 performed best (after post-processing) on at least one of the 40 problems considered in the study. These 11 combinations included 5 of the 6 dispatch rules (here, WSPT was never best) and all 3 release policies.

Table 1 Characteristics of the eight problem sets

\begin{tabular}{|c||c|c|c|}
\hline Problem Set & Number of Bottlenecks & Avg. Due Date & Due Date Range \\
\hline \hline 1 & 1 & loose & wide \\
\hline 2 & 1 & loose & narrow \\
\hline 3 & 1 & tight & wide \\
\hline 4 & 1 & tight & narrow \\
\hline 5 & 2 & loose & wide \\
\hline 6 & 2 & loose & narrow \\
\hline 7 & 2 & tight & wide \\
\hline 8 & 2 & tight & narrow \\
\hline
\end{tabular}

The results reported below were obtained on a suite of 40 scheduling problems similar to the ones described in [24]. The series consisted of eight sets of scheduling problems obtained by adjusting three parameters to cover a wide range of scheduling conditions (See Table 1): an average due date parameter (tight versus loose average due date), a due date range parameter (narrow versus wide range of due dates), and a parameter controlling the number of major bottlenecks (in this case one or two). For each parameter combination, a set of 5 scheduling problems was randomly generated, thereby resulting in a total of 40 problems ( 5 problems x 2 average due date values $\mathrm{x}$ 2 due date ranges $\mathrm{x} 2$ bottleneck configurations). Each problem involved 20 jobs and 5 resources for a total of 100 operations. Marginal tardiness costs in these problems were set to be, on average, ten times larger than marginal inventory costs to model a situation where tardiness costs dominate but inventory costs are non-negligible ${ }^{5}$.

The SA procedure was run 10 times on each problem. For each problem, we recorded both the average performance of the procedure (referred to below as SA-AVG) as well as the best solution it found for each problem over 10 runs (SA-BEST). In each run,

\footnotetext{
${ }^{5}$ Similar results have also been obtained on a set of problems where marginal tardiness costs were on average five times larger than marginal inventory costs.
} 
the initial temperature, $T_{0}$, was set to 700 , temperature $T_{1}$ was 6.25 and the cooling rate $\alpha$ was 0.85 . The value of $\beta$ was $1000{ }^{6}$. The number $N$ of iterations in the inner-loop of the procedure (See Figure 1) was set to 300,000 .

Fig. 5: Comparison of SA and a combination of 39 dispatch rules and release policies with and without optimal idle time insertion.

Figure 5 compares the schedules produced by the SA procedure with the best

\footnotetext{
${ }^{6}$ The problems that were run typically had optimal solutions with a value ranging between 3000 and 15000 . Setting $\beta$ to 1000 was sufficient to guarantee that all precedence constraints were satisfied at the end of each run.
} 
schedules obtained on each problem by the 39 combinations of dispatch rules and release policies both with and without idle time optimization. For instance, on Problem Set 6 (problems with two bottleneck resources, loose average due dates and narrow due date ranges), (1) SA-BEST reduced schedule cost by almost $11 \%$ compared to SA-AVG, (2) SA-AVG reduced schedule cost by about $18 \%$ compared to the 39 combinations of dispatch rules and release policies with optimal idle time insertion and (3) performance of the 39 combinations of dispatch rules and release policies (taking the best of 39 schedules on each problem) improves by more than $6 \%$ with optimal idle time insertion.

Overall, Figure 5 indicates that SA-BEST consistently outperforms the combinations of dispatch rules and release policies with and without idle time insertion on all 8 problem sets. The comparison also holds for SA-AVG with the exception of the two easier problem sets (Problem Set 1 and 5, i.e. problems with loose and widely spread due dates), where SA-AVG does slightly worse than the 39 combinations with idle time optimization. Notice that SA-AVG still outperforms the 39 combinations without idle time optimization on these two problem sets. Overall, compared against the 39 combinations of dispatch rules and release policies without idle time optimization, SA-AVG reduced schedule cost by close to $16 \%$ and SA-BEST by close to $28 \%$. Even when, on each problem, idle time was optimally inserted in each of the 39 schedules obtained by the combinations of dispatch rules and release policies, SA-AVG still reduced schedule cost by an average of about $7 \%$ and SA-BEST by over $20 \%$. A more detailed analysis indicates that these reductions in schedule cost reflect reductions in both tardiness and inventory costs. However, while running all 39 combinations of dispatch rules and release policies requires only a few CPU seconds on each problem and about 45 to 50 CPU seconds when idle time is optimally inserted in each of the 39 schedules, a SA 
run takes 3 to 5 minutes on a DECstation 5000/200 running C.

Fig. 6 Performance of the SA procedure with and without idle time optimization.

Additional experiments were also conducted to evaluate the performance of the $\mathrm{SA}$ procedure with respect to idle time optimization. Figure 6 summarizes these experiments, reporting both the average and best performance of the SA procedure over 10 runs with and without post-processing for optimal idle time insertion. The results clearly indicate that the schedules produced by the SA procedure are nearly 
optimal with respect to idle time insertion, thereby validating the choice of the LEFTand RIGHT-SHIFT operators used to define the neighborhood of the procedure. On average, idle time insertion improved performance of SA-BEST by a meager $0.94 \%$ (with a standard deviation of $0.8 \%$ ) and that of $\mathrm{SA}-\mathrm{AVG}$ by $1.02 \%$ (with a standard deviation of $0.5 \%$ ).

The results in Figure 5 and 6 generally attest to the ability of the SA procedure to produce high quality solutions, often significantly reducing schedule cost compared to other well-regarded scheduling heuristics. They also indicate that the computational requirements of the procedure are quite large compared to these other heuristics, though experiments with larger problems suggest that the average complexity of our SA procedure only grows linearly with the size of the problem.

Finally, we observe that the performance of the SA procedure can significantly vary from one run to another, as illustrated by the results in Figure 5. In our experiments, an average run of SA produced schedules with costs $14 \%$ higher than those of the best schedule obtained over 10 runs (SA-AVG vs. SA-BEST). This suggests that important speedups could possibly be obtained if the procedure was more consistent in producing high quality solutions. In the following section, a meta-heuristic procedure is presented that aims at reducing performance variability using artificial costs to dynamically focus the SA procedure on critical subproblems.

\section{$5 \quad$ Focused Simulated Annealing Search}

Figure 7 depicts 5 typical runs of the SA procedure introduced in the previous sections, plotting the cost of the best schedule found in each run, as the the temperature is slowly 
lowered over time.

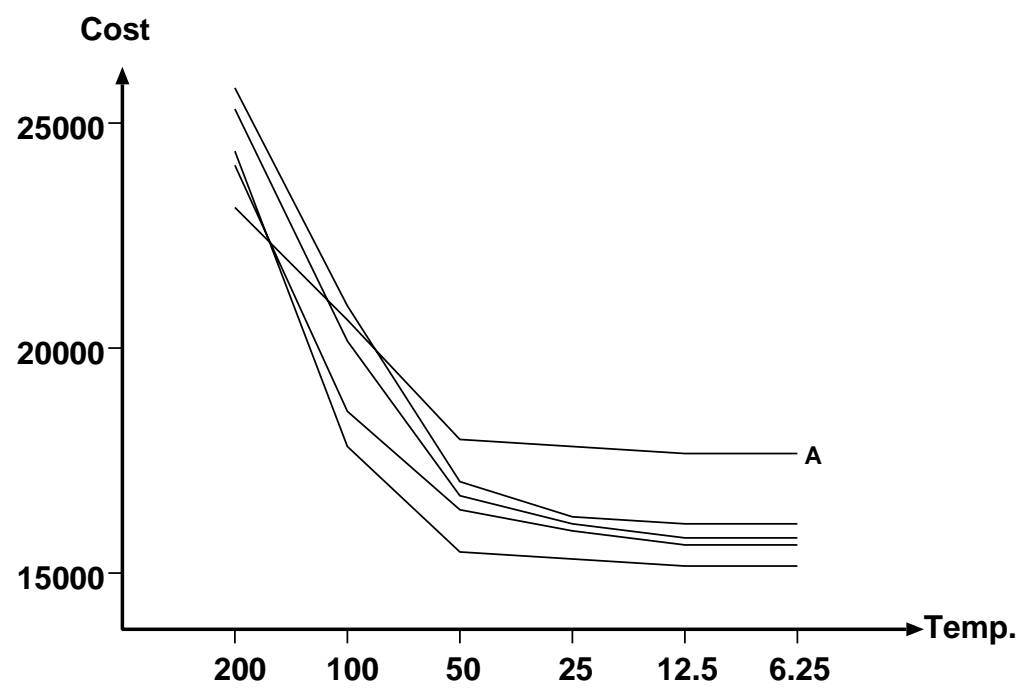

Fig. 7 Solution improvement in 5 runs of SA.

The behavior exhibited in Figure 7 is characteristic of SA search procedures: the largest improvements are observed at relatively high temperatures. In the case of our SA procedure, we observed that below $T=50$ the quality of the solution never improved by more than a few percent. In other words, the early stage of the procedure is the one that determines whether or not the procedure will get trapped in a local minimum (e.g. See run A in Figure 7). The remainder of this section describes a meta-heuristic that relies on the dynamic introduction of artificial costs in the objective function to focus SA on critical subproblems and attempt to steer clear of local minima during the high temperature phase of the procedure. Below we refer to the resulting procedure as "Focused Simulated Annealing" (FSA) search.

To improve the quality of an existing solution, FSA iteratively identifies major inefficiencies in the current solution and attempts to make these inefficiencies more obvious to the search procedure by artificially inflating their costs. As a result, the search procedure works harder on getting rid of these inefficiencies, possibly introducing new inefficiencies in the process. By regularly tracking sources of inefficiency in the existing solution and reconfiguring the cost function to eliminate these inefficiencies, FSA can increase the chances that the procedure finds a high quality solution. 


$$
\begin{aligned}
& T=T_{0} ; \\
& x=x_{0}(\in S) \\
& \text { BestSol }=x_{0} ; M=\operatorname{cost}(\text { BestSol }) \text {; } \\
& \text { while }\left(T>T_{1}\right) \quad\{ \\
& \text { if }\left(T>T_{2}\right) \\
& \text { CritSubp =identify-high-cost-subp }(x) \text {; } \\
& \text { else } \\
& \text { CritSubp }=\emptyset \\
& \text { for } i=1, N \quad\{ \\
& x^{\prime}=\text { neighbor }(x) \text {; } \\
& \text { if }\left(\operatorname{cost} 1\left(x^{\prime}\right)<\operatorname{cost} 1(x)\right) \quad x=x^{\prime} \\
& \text { else if }\left(\operatorname{rand}()<\exp \left\{\left(\operatorname{cost} 1(x)-\operatorname{cost} 1\left(x^{\prime}\right)\right) / T\right\}\right) \quad x=x^{\prime} \text {; } \\
& \text { \} } \\
& \text { if }\left(\operatorname{cost}\left(x^{\prime}\right)<M\right) \quad\left\{\text { BestSol }=x^{\prime} ; M=\operatorname{cost}(\text { BestSol }) ;\right\} \\
& \text { if ( } M \text { was not modified in the above loop) } T=T * \alpha \text {; }
\end{aligned}
$$

Fig. 8 The FSA Procedure: A meta-heuristic that continuously attempts to reduce major inefficiencies in the solution.

Pseudo-code for the FSA procedure is given in Figure $8 . T_{2}$ is a threshold temperature between $T_{0}$ and $T_{1}$. Below $T_{2}$, FSA behaves exactly as the SA search procedure described in Figure 1. Before reaching this temperature, the procedure uses a different cost function to decide whether or not to accept transitions to neighboring solutions, namely:

$$
\operatorname{cost} 1(x)=\operatorname{cost}(x)+\operatorname{ArtifCost}(x)
$$

where:

$$
\operatorname{ArtifCost}(x)=\sum_{O_{i}^{l} \in C_{r i t S u b p}}\left\{k\left(t \cos t_{i}^{l}+i \cos t_{i}^{l}\right)\right\}
$$

or, equivalently:

$$
\operatorname{cost} 1(x)=\sum_{l \in J} \sum_{1 \leq i \leq n_{l}}\left(f \operatorname{cost}_{i}^{l}+t \cos t_{i}^{l}+i \cos t_{i}^{l}\right)+\sum_{O_{i}^{l} \in C r i t \text { Subp }}\left\{k\left(t \cos t_{i}^{l}+i \cos t_{i}^{l}\right)\right\}
$$

$k$ is a parameter that controls the amount by which the costs associated with operations in critical subproblems are inflated. In our experiments, we found that setting $k$ to 2 and $T_{2}$ to 50 generally yielded good results. Results obtained with other values for these parameters are provided in Appendix B. 
Notice that inflating the costs associated with one or several subproblems is equivalent to reducing the temperature associated with the corresponding components of the objective function (or raising the temperature in the remainder of the problem). Accordingly, FSA can be viewed as a SA procedure in which transition probabilities are subject to different temperatures in different parts of the problem. Temperatures in different subproblems are regularly modified (lowered or raised) to get rid of major inefficiencies in one part or another of the working solution. In this regard, FSA is reminiscent of the Strategic Oscillation idea of developing non-monotonic cooling schedules [7, 21, 10]. However, while Strategic Oscillation cooling schedules proposed in the literature vary temperature in the entire problem, FSA emphasizes selective temperature variations in dynamically identified subproblems, as detailed below.

The specific parts of the solution in which FSA attempts to eliminate inefficiencies are determined by the identify-high-cost-subp() function. Here several variations of the procedure are considered that differ in the way they decompose the problem: a job-based variation, a resource-based variation and an operation-based variation.

"Critical Job" (CJ) variation This variation of FSA dynamically inflates the costs associated with critical jobs. Here, identify-high-cost-subp() computes the the cost of each job $j_{l}$ in the current schedule, namely,

$$
\sum_{1 \leq i \leq n_{l}}\left(t \cos t_{i}^{l}+i \cos t_{i}^{l}\right)
$$

. The function then returns the set of all jobs whose costs are above $p \cdot a v_{R}$, where $a v_{R}$ is the average cost of a job in the current schedule and $p$ is a constant. In the experiments reported below, $p$ was empirically set to 3 . Below we refer to this variation of the procedure as $F S A(C J)$. Results obtained with other values of $p$ are also reported in Appendix B.

"Bottleneck Resource" (BR) variation This variation of FSA inflates the costs associated with critical ("bottleneck") resources in the existing schedule. This is done by computing a cost for each resource $R_{k}$ :

$$
\sum_{R_{i}^{l}=R_{k}}\left(t \cos t_{i}^{l}+i \cos t_{i}^{l}\right)
$$

In this case, the highest cost resource is selected and all the operations requiring this resource are returned by identify-high-cost-subp(). This procedure will be referred to 
as $F S A(B R)$.

"Critical Operation" (CO) variation Here, FSA focuses on critical operations rather than critical jobs or critical resources. The average cost of an operation in the current schedule, $a v_{O}$, is computed:

$$
a v_{O}=\sum_{l \in J} \sum_{1 \leq i \leq n_{l}}\left(t \operatorname{cost}_{i}^{l}+i \operatorname{cost}_{i}^{l}\right) / \sum_{l \in J} n_{l}
$$

All the operations with a cost above $q \cdot a v_{O}$ are considered critical. $q$ is a constant. In the experiments reported below, $q$ was equal to 3 . We will denote this procedure $F S A(C O)$. Results obtained with other values of $q$ are also reported in Appendix B.

\section{Performance Evaluation}

To evaluate the effectiveness of FSA, all three variations of the procedure were run on a set of 40 scheduling problems similar to the ones described in Section 4. Each variation was run 10 times on each problem. Table 2 compares each of the three variations of the FSA procedure against the SA procedure described in Section 3. Both the average and best performance over 10 runs are reported.

Table 2 Cost Reduction (\%) obtained by FSA over SA

\begin{tabular}{|c||c|c||c|c||c|c|}
\hline \multicolumn{1}{|c||}{$\begin{array}{c}\text { Problem } \\
\text { Set }\end{array}$} & \multicolumn{2}{c||}{ FSA(CJ) } & \multicolumn{2}{c||}{ FSA(BR) } & \multicolumn{2}{c|}{ FSA(CO) } \\
\cline { 2 - 7 } & Avg & Best & Avg & Best & Avg & Best \\
\hline \hline 1 & 4.5 & 2.0 & -0.8 & 0.9 & -0.6 & -0.6 \\
\hline 2 & 6.5 & 5.1 & 4.3 & 2.3 & 3.5 & 4.3 \\
\hline 3 & 7.3 & 5.2 & -1.2 & -2.6 & -0.9 & -2.3 \\
\hline 4 & 0.4 & 0.2 & -2.4 & -2.2 & -2.1 & -2.2 \\
\hline 5 & 8.6 & 4.5 & -9.4 & -1.7 & 1.9 & 3.2 \\
\hline 6 & 8.0 & 2.4 & -2.8 & -4.3 & 2.3 & 4.2 \\
\hline 7 & 6.7 & 6.2 & -25.2 & -6.3 & -2.4 & 0.4 \\
\hline 8 & 0.2 & 3.5 & -2.7 & -0.5 & -2.1 & 0.7 \\
\hline \hline Overall & 5.2 & 3.6 & -5.0 & -2.0 & -0.5 & 0.9 \\
\hline
\end{tabular}

The results in Table 2 show that the dynamic introduction of artificial costs, as implemented in the FSA procedure, can potentially lead to significant improvements both in the average and best performance of the SA procedure. The results also show that the effectiveness of this approach depends on the type of subproblems considered 
by the procedure. While FSA(CJ) reduced schedule cost by an average of $5.2 \%$ and improved the quality of the best schedule found in 10 runs by an average of $3.6 \%$, the other two variations of the procedure, FSA(BR) and FSA(CO), did not fare as well. FSA(CO) performed approximately like the original SA procedure and FSA(BR) actually did worse. Below, we further analyze the performance improvement obtained with FSA(CJ). In the following section, we attempt to explain why FSA(CO) and FSA(BR) did not perform as well. For now, we further analyze the performance improvements observed with FSA(CJ).

Figure 9 and 10 show the cost distributions of the schedules obtained by successively running SA and FSA(CJ) 300 times on two typical scheduling problems.
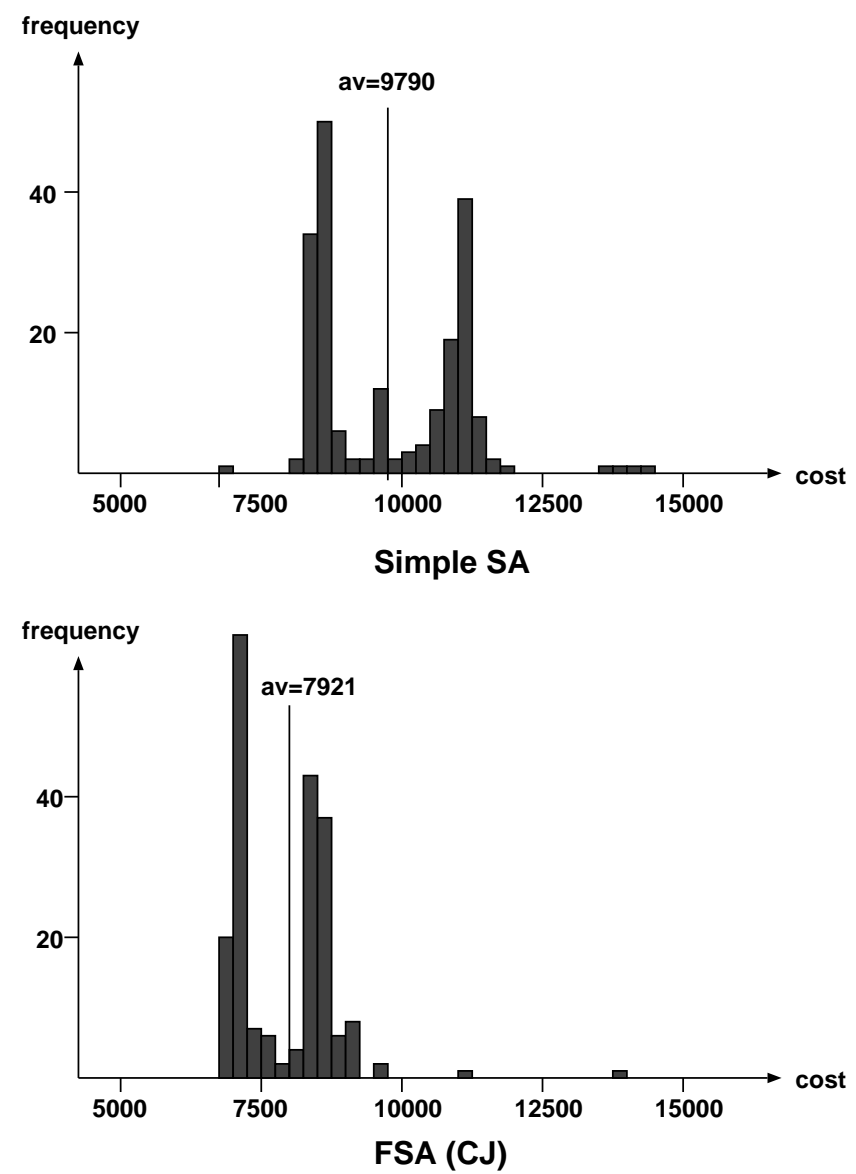

Fig. 9 Improvement of FSA(CJ) over the original SA procedure (Problem 1)

On the problem in Figure 9, the improvement obtained with FSA(CJ) is quite obvious: both the average and standard deviation of the cost distribution produced by 
FSA(CJ) are lower than those of the original SA procedure. Accordingly, it appears that for this problem the probability of getting trapped in a local optimum has been greatly reduced. This in turn can translate in significant reductions in computation time. For instance, while the original SA procedure would require an average of 2.5 runs to find a schedule with cost below 9000 , FSA(CJ) would only require an average of 1.1 run, a saving of more than $50 \%$. To find a schedule of cost below 8000, FSA(CJ) reduces computation time by more than $90 \%$.

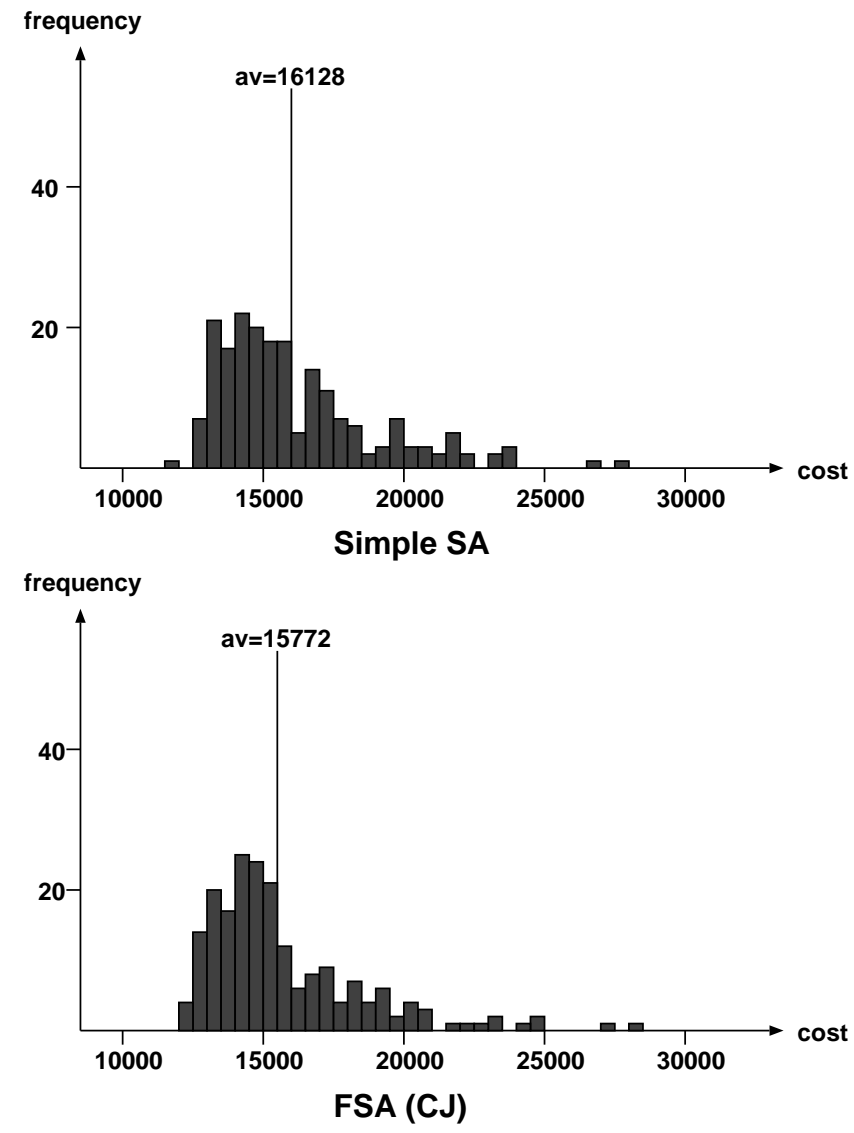

\section{Fig. 10 Improvement of FSA(CJ) over the original SA procedure (Problem 2)}

On the other hand, for the problem in Figure 10, the performance improvement yielded by FSA(CJ) is rather modest: no significant reduction in average schedule cost or even in the standard deviation of the distribution.

Looking more carefully at these two problems, we observe that, in the case of the problem in Figure 9, SA yields a cost distribution with two clearly separated peaks, 
thereby suggesting that the procedure is often caught in local minima. In contrast, in the case of the problem analyzed in Figure 10, the cost distribution obtained using the original SA procedure is generally more compact. This would suggest that FSA(CJ) is more effective in those situations where the original procedure is more likely to get trapped in local optima.

To verify this hypothesis, we measure for each problem the average reduction in schedule cost yielded by FSA(CJ) (compared to the original SA procedure) and the spread of the cost distribution obtained with the original SA procedure. This spread is simply measured as the standard deviation of the cost distribution obtained by $\mathrm{SA}$ divided by the mean of this distribution. The results for all 40 problems of the study are summarized in Figure 11.

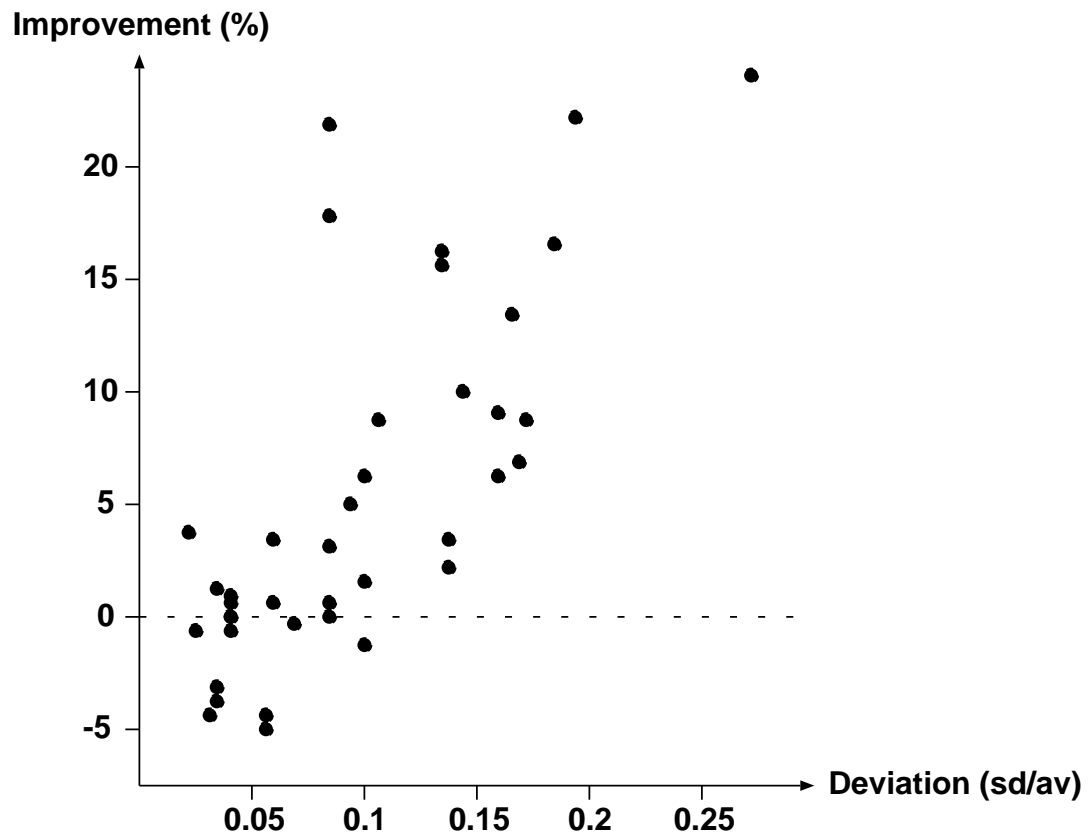

Fig. 11 Improvements obtained with $\operatorname{FSA}(\mathrm{CJ})$ as a function of the relative variation in schedule cost observed when using the original SA procedure.

The graph clearly confirms our intuition. The most important improvements are observed on problems where the original SA procedure showed the least consistency, namely those problems where it had the highest chance of getting trapped in local minima. The Figure also indicates that FSA(CJ) rarely performs worse than the original $\mathrm{SA}$ procedure, and, when it does, the degradation in schedule quality is marginal. 


\section{Further Analysis}

If we are to apply FSA to other problems, we need to understand why some variations of the procedure perform better than others. There are at least two ways of approaching this question. One approach is to attempt to analyze the search procedure and the neighborhood structure it relies on and try to understand how the choice of a given type of subproblems influences the effectiveness of FSA on this specific class of scheduling problems. This approach is probably the one a scheduling expert would be tempted to follow. It could potentially lead to very insightful conclusions for the class of scheduling problems of interest in this study. However, our purpose here is different, as we are looking for insight that can possibly carry over to other domains. For this reason, we take a different approach and limit our analysis to the external behavior of the search procedure.

As pointed out at the beginning of Section 5, the early phase of a SA run, where temperature is still high, generally determines whether the procedure gets caught in a local minimum or not. Different neighborhood structures for a same class of problem can possibly lead to different types of local minima. The nature of these local minima can in turn affect the effectiveness of different problem decompositions in the FSA procedure. In Figure 12, we analyze cost reductions in different types of subproblems during the lower temperature phase of the original SA procedure. Specifically, Figure 12 considers improvements in three different types of subproblems:

1. CJ: the set of critical jobs that would be identified by FSA(C.J) at $T=100$

2. BR: the critical ("bottleneck") resource that would be used by FSA(BR) at $T=$ 100

3. CO: the set of critical operations that would be considered by FSA(CO) at $T=$ 100 


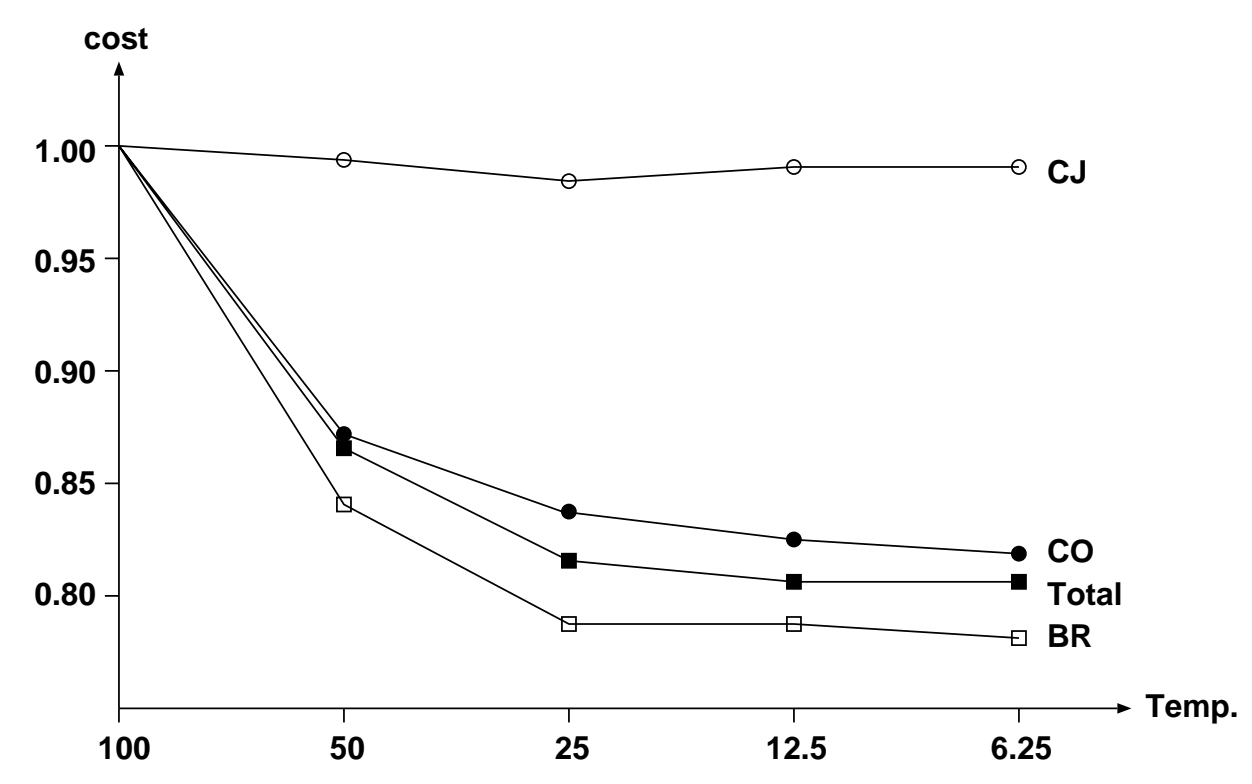

Fig. 12 Changes of Cost in the Later Stage of SA

For each of these subproblems, Figure 12 plots the average variation in cost associated with these 3 subproblems as the temperature in the original SA procedure is progressively lowered. The curve labeled "Total" plots the cost variations of the overall schedule as temperature decreases. The points in Figure 12 represent averages taken over the set of 40 problems studied in Section 6 and over 10 runs of SA on each problem.

Figure 12 indicates that, when using the original $\mathrm{SA}$ procedure, major inefficiences in job schedules do not get corrected below temperature $T=100$, while major inefficiencies at the level of critical resources or critical operations are still easy to eliminate. This explains why FSA(CJ) is the variation that performs best: it is the one that best matches the weaknesses of the original SA procedure. By working hard on eliminating inefficiencies at the level of critical jobs, FSA(CJ) reduces the chances that such inefficiencies remain when the procedure reaches its lower temperature phase, a phase when it is no longer effective at getting rid of these inefficiencies. For the same reason, the BR curve suggests that FSA(BR) wastes its time getting rid of inefficiencies that are still easy to eliminate in the lower temperature phase of the procedure, and hence can be expected to perform poorly, as observed in the results presented in Section 6 . 


\section{Summary and Concluding Remarks}

In summary, the contribution of this work is twofold:

1. On the scheduling front, a SA procedure has been developed to solve job shop scheduling problems with both tardiness and inventory costs. The procedure has been shown to produce high quality solutions, reducing schedule cost by $28 \%$ over a combination of 39 well-regarded dispatch rules and release policies (and by $20 \%$ when the dispatch schedules are post-processed for optimal idle time insertion), though at the expense of significant computational efforts.

2. To reduce the computational requirements of this procedure, a meta-heuristic search procedure called Focused Simulated Annealing (FSA) search has been developed. This procedure aims at reducing variability in the performance of SA by dynamically focusing on the elimination of major inefficiencies in the solution. The procedure works by dynamically inflating the costs associated with critical subproblems and requires a decomposable objective function.

Three variations of FSA have been developed for the job shop scheduling problem with tardiness and inventory costs. These variations of the procedure differ in the type of subproblems they rely on: job subproblems, resource subproblems, or operation subproblems. Experiments show that, with the right decomposition, FSA can significantly improve solution quality especially on problems where search is likely to get caught in local minima. Equivalently, for the same solution quality, FSA can greatly reduce computation time over a regular SA search.

Our experiments also indicate that the performance of FSA critically depends on the selection of a good decomposition of the objective function. An analysis suggests that the most effective decompositions are those corresponding to subproblems whose solutions are particularly difficult to improve during the low temperature phase of the SA procedure. By focusing on inefficiencies at the level of these subproblems, FSA can greatly reduce the chance of getting trapped in local minima.

As is often the case in this type of study, many design alternatives remain to be explored. Further work will also be required to assess the effectiveness of FSA or FSA-like meta-heuristics in combination with more sophisticated SA procedures, e.g. 
procedures incorporating some aspects of Tabu Search [9, 25, 19]. Like Strategic Oscillation [7, 21, 10], FSA can be viewed as implementing a non-monotonic cooling schedule, though selectively, by focusing on dynamically identified subproblems. Strategic Oscillation could possibly also be exploited to control the value of $\beta$, the parameter used in our procedure to penalize precedence constraint violations within a job. Other aspects of Tabu Search such as Target Analysis [8, 15], which, like FSA, adds a term to the objective function to drive the procedure towards high quality solutions, would also be worth comparing with and possibly incorporating in the existing procedure.

\section{Acknowledgements}

This research was supported, in part, by the Defense Advanced Research Projects Agency under contract F30602-91-C-0016 and, in part, by an industrial grant from NEC. The research was carried out while the second author was a visiting scientist at Carnegie Mellon's Robotics Institute.

\section{References}

[1] Baker, K.R. "Introduction to Sequencing and Scheduling," Wiley, 1974.

[2] Cerny, V., “Thermodynamical Approach to the Traveling Salesman Problem: An Efficient Simulation Algorithm," J. Opt. Theory Appl., Vol. 45, pp. 41-51, 1985.

[3] French, S. "Sequencing and Scheduling: An Introduction to the Mathematics of the Job-Shop," Wiley, 1982.

[4] Fry,T., R. Amstrong and J. Blackstone "Minimizing Weighted Absolute Deviation in Single Machine Scheduling," IIE Transactions, Vol. 19, pp. 445-450, 1987.

[5] Garey, M. R. and Johnson, D. S. "Computers and Intractability: A Guide to the Theory of NP-Completeness," Freeman and Co., 1979

[6] Garey M.R., R.E. Tarjan, and G. T. Wilfgong, "One-Processor Scheduling with Symmetric Earliness and Tardiness Penalties," Mathematics of Operations Research, Vol. 13, No. 2, pp. 330-348, 1988.

[7] Glover, F. "Heuristics for Integer Programming Using Surrogate Constraints," Decision Sciences, Vol. 8, No. 1, pp. 156-166, January 1977. 
[8] Glover, F. "Future Paths for Integer Programming and Links to Artificial Intelligence," Computer and Operations Research, Vol. 13, No. 5, pp. 533-549, 1986.

[9] Glover, F. "Tabu Thresholding: Improved Search by Non-Monotonic Trajectories," Technical Report, Graduate School of Business, University of Colorado, Boulder, Colorado 80309-0419, 1993.

[10] Glover, F. and M. Laguna "Tabu Search," Chapter in Modern Heuristic Techniques for Combinatorial Problems, pp. 70-150, Colin Reeves (Ed.), Blackwell Scientific Publications, Oxford, 1992

[11] Holland J. "Adaptation in Natural and Artificial Systems" University of Michigan Press, Ann Arbor, MI. 1975.

[12] Johnson, D. S., Aragon, C. R., McGeoch, L. A. and Schevon, C. "Optimization by Simulated Annealing: Experimental Evaluation; Part I, Graph Partitioning" Operations Research Vol. 37 no. 6, pp. 865-892, 1989

[13] Johnson, D. S., Aragon, C. R., McGeoch, L. A. and Schevon, C. "Optimization by Simulated Annealing: Experimental Evaluation; Part II, Graph Coloring and Number Partitioning" Operations Research Vol. 39 no. 3, pp. 378-406, 1991

[14] Kirkpatrick, S., Gelatt, C. D., and Vecchi, M. P., "Optimization by Simulated Annealing," Science, Vol. 220, pp. 671-680, 1983.

[15] Laguna M. and F. Glover "Integrating Target Analysis and Tabu Search for Improved Scheduling Systems," Expert Systems with Applications, Vol. 6, pp. 287$297,1993$.

[16] Matsuo H., C.J. Suh, and R.S. Sullivan "A Controlled Search Simulated Annealing Method for the General Jobshop Scheduling Problem" Tech. Report, "Dept. of Management, The Univ. of Texas at Austin Austin, TX, 1988.

[17] Morton, T.E. “SCHED-STAR: A Price-Based Shop Scheduling Module," Journal of Manufacturing and Operations Management, pp. 131-181, 1988.

[18] Morton, T.E. and Pentico, D.W. "Heuristic Scheduling Systems," Wiley Series in Engineering and Technology Management, 1993 
[19] Nowicki E. and C. Smutnicki "A Fast Taboo Search Algorithm for the Job Shop Problem," Technical University of Wroclaw, Institute of Engineering Cybernetics, ul. Janiszewskiego 1/17, 50-372 Wrocklaw, Poland, 1993.

[20] Osman,I.H., and Potts, C.N., "Simulated Annealing for Permutation Flow-Shop Scheduling," OMEGA Int. J. of Mgmt Sci., Vol. 17, pp. 551-557, 1989.

[21] Osman, I.H. "Meta-Strategy Simulated Annealing and Tabu Search Algorithms for the Vehicle Routing Problem" Annals of Operations Research, Vol. 41, pp. 421451, 1993.

[22] Ow, P.S. and T. Morton, "The Single Machine Early/Tardy Problem" Management Science, Vol. 35, pp. 177-191, 1989.

[23] Sadeh, N. "Look-ahead Techniques for Micro-Opportunistic Job Shop Scheduling" Ph.D. thesis, School of Computer Science, Carnegie Mellon University, Pittsburgh, PA 15213, March 1991.

[24] Sadeh, N. "Micro-Opportunistic Scheduling: The Micro-Boss Factory scheduler" Ch. 4, Intelligent Scheduling, Zweben and Fox (Ed.), Morgan Kaufmann Publishers, 1994.

[25] Taillard, E. "Parallel Taboo Search Technique for the Job Shop Scheduling Problem," ORSA Journal on Computing, Vol. 6, pp. 108-117, 1994.

[26] Van Laarhoven, P.J., Aarts, E.H.L., and J.K. Lenstra "Job Shop Scheduling by Simulated Annealing," Operations Research, Vol. 40, No. 1, pp. 113-125, 1992.

[27] Vepsalainen,A.P.J. and Morton, T.E. "Priority Rules for Job Shops with Weighted Tardiness Costs," Management Science, Vol. 33, No. 8, pp. 1035-1047, 1987.

\section{Appendix A: Idle Time Insertion as a Linear Program}

The problem of optimally inserting idle in an existing job shop schedule (i.e. given completely defined operation sequences on each resource) can be formulated as a linear program, as detailed below:

$$
M I N \sum_{l \in J}\left\{\operatorname{tard}_{l} \cdot \tau_{l}+\sum_{i=1}^{n_{l}-1}\left[i n v_{i}^{l} \cdot\left(s t_{i+1}^{l}-s t_{i}^{l}\right)\right]+i n v_{n_{l}}^{l} \cdot\left(d u_{n_{l}}^{l}+\epsilon_{l}\right)\right\}
$$


such that:

$$
\begin{array}{ll}
\tau_{l}-\epsilon_{l}-s t_{n_{l}}^{l}=d u_{n_{l}}^{l}-d d_{l} & l=1, \ldots, n \\
s t_{i}^{l}-s t_{i+1}^{l} \leq-d u_{i}^{l} & l=1, \ldots, n \quad i=1, \ldots, n_{l}-1 \\
s t_{l o w(k, j)}^{u p(k, j)}-s t_{l o w(k, j+1)}^{u p(k, j+1)} \leq-d u_{l o w(k, j)}^{u p\left(R_{k}, j\right)} & k=1, \ldots, m \quad j=1, \ldots, p_{k}-1 \\
\tau_{l}, \epsilon_{l} \geq 0 & l=1, \ldots, n \\
s t_{1}^{l} \geq e r d_{l} & l=1, \ldots, n \\
s t_{n_{l}}^{l} \leq l c d_{l}-d u_{n_{l}}^{l} & l=1, \ldots, n
\end{array}
$$

where:

- $\tau_{l}$ is the tardiness of job $j_{l}$

- $\epsilon_{l}$ is the earliness of job $j_{l}$

- $O_{l o w(k, j)}^{u p(k, j)}$ is the $\mathrm{j}$-th operation scheduled on resource $R_{k}$ (in the given schedule). In other words, $u p(k, j)$ is the index of the job to which this opeation belongs and $\operatorname{low}(k, j)$ the index of this operation within its job

- $p_{k}$ is the number of operations requiring resource $R_{k}$

- The other notations are as defined in Section 2

Note that, in Equation (2), when job $j_{l}$ is tardy, $\tau_{l}=s t_{n_{l}}^{l}+d u_{n_{l}}^{l}-d d_{l} \geq 0$ and $\epsilon_{l}=0$, and, when it is early, $\epsilon_{l}=d d_{l}-\left(s t_{n_{l}}^{l}+d u_{n_{l}}^{l}\right) \geq 0$ and $\tau_{l}=0$. A similar formulation was first proposed by Fry et al. for the one-machine early/tardy problem [4]. While more efficient procedures are described in the literature for the one-machine early/tardy problem, including an $O(N \log N)$ procedure developed by Garey et al. [6], it is not clear at this time how these procedures could be efficiently generalized to the job shop case.

\section{Appendix B: Results Obtained Under Different Parameter Settings}

This appendix summarizes results obtained with FSA for different values of the following four parameters: 
- $T_{2}$ : temperature above which FSA artificially inflates the costs of critical subproblems. The results in Table 3 were obtained using $F S A(C J)$, the variation of FSA that performed best in our experiments. In these experiments, $k=2$ and $p=3$.

- $k$ : the parameter by which FSA inflates the costs of critical subproblems. The results in Table 4 were obtained using FSA(CJ), the variation of FSA that performed best in our experiments. In these experiments, $T_{2}=50$ and $p=3$.

- $p$ : the parameter used by $F S A(C J)$ to identify critical jobs. Results obtained with different values of this parameter are summarized in Table 5. In these experiments, $T_{2}=50$ and $k=2$.

- q: the parameter used by $F S A(C O)$ to identify critical operations. Results obtained with different values of this parameter are summarized in Table 6 . In these experiments, $T_{2}=50$ and $k=2$.

More detailed definitions of these parameters are provided in Section 5. The tables below report both average and best performance of FSA over 10 runs.

The best results are generally obtained for $T_{2}=50, k=2, p=3$ and $q=3$, the values used in the experiments reported in Section 6 . 
Table 3 Percentage performance improvement $(+) /$ degradation(-) observed when running FSA(CJ) with different values of $T_{2}$. Performance with $T_{2}=50$ is used as the reference.

\begin{tabular}{|c||c|c|c|c||c|c|c|c|}
\hline \multicolumn{1}{|c||}{ Problem } & \multicolumn{4}{c||}{ Best Performance } & \multicolumn{4}{c|}{ Average Performance } \\
\cline { 2 - 9 } & $T_{2}=25$ & $T_{2}=50$ & $T_{2}=100$ & $T_{2}=200$ & $T_{2}=25$ & $T_{2}=50$ & $T_{2}=100$ & $T_{2}=200$ \\
\hline \hline 1 & -2.3 & 0.0 & -7.7 & -3.5 & -2.4 & 0.0 & -1.2 & -0.2 \\
\hline 2 & -0.3 & 0.0 & 3.3 & 1.6 & 0.8 & 0.0 & 0.2 & -4.1 \\
\hline 3 & -2.3 & 0.0 & 0.5 & 0.0 & -2.4 & 0.0 & -0.5 & 1.3 \\
\hline 4 & -3.7 & 0.0 & -1.6 & -0.9 & -2.5 & 0.0 & -0.7 & 0.7 \\
\hline 5 & -1.2 & 0.0 & 0.0 & 0.8 & -0.1 & 0.0 & -2.6 & -1.2 \\
\hline 6 & -1.8 & 0.0 & 2.5 & -0.5 & -1.1 & 0.0 & 1.4 & 1.7 \\
\hline 7 & -0.6 & 0.0 & -1.2 & -4.0 & -3.6 & 0.0 & -0.1 & -0.4 \\
\hline 8 & -2.1 & 0.0 & -5.6 & -6.8 & -0.4 & 0.0 & 0.7 & 2.8 \\
\hline \hline Overall & -1.8 & 0.0 & -1.2 & -1.7 & -1.5 & 0.0 & -0.4 & 0.1 \\
\hline
\end{tabular}


Table 4 Percentage performance improvement(+)/degradation(-) observed when running FSA(CJ) with different values of $k$. Performance with $k=2$ is used as the reference.

\begin{tabular}{|c||c|c|c|c||c|c|c|c|}
\hline \multirow{2}{*}{\multicolumn{1}{|c||}{ Problem }} & \multicolumn{3}{c||}{ Best Performance } & \multicolumn{3}{c|}{ Average Performance } \\
\cline { 2 - 8 } & $k=1$ & $k=2$ & $k=3$ & $k=4$ & $k=1$ & $k=2$ & $k=3$ & $k=4$ \\
\hline \hline 1 & -3.8 & 0.0 & -4.4 & -0.3 & 1.0 & 0.0 & 0.8 & 0.1 \\
\hline 2 & -0.2 & 0.0 & -1.2 & -2.0 & 1.3 & 0.0 & 0.0 & -1.3 \\
\hline 3 & -1.9 & 0.0 & -2.6 & 3.2 & -2.2 & 0.0 & 1.6 & 1.5 \\
\hline 4 & -2.2 & 0.0 & -3.4 & -3.6 & 0.4 & 0.0 & -2.4 & -1.1 \\
\hline 5 & -3.6 & 0.0 & -2.8 & -0.5 & -1.1 & 0.0 & -4.2 & -12.1 \\
\hline 6 & -0.3 & 0.0 & 2.5 & -0.9 & -1.1 & 0.0 & -2.4 & -1.8 \\
\hline 7 & 2.9 & 0.0 & -5.2 & 2.1 & 3.2 & 0.0 & 0.1 & -3.1 \\
\hline 8 & -3.6 & 0.0 & -2.1 & -5.0 & 2.9 & 0.0 & 3.0 & 2.5 \\
\hline \hline Overall & -1.6 & 0.0 & -2.4 & -0.9 & 0.6 & 0.0 & -0.4 & -1.9 \\
\hline
\end{tabular}


Table 5 Percentage performance improvement $(+) /$ degradation(-) observed when running FSA(CJ) with different values of $p$. Performance with $p=3$ is used as the reference.

\begin{tabular}{|c||c|c|c|c||c|c|c|c|}
\hline \multirow{2}{*}{ Problem } & \multicolumn{3}{||}{ Best Performance } & \multicolumn{3}{c||}{ Average Performance } \\
\cline { 2 - 8 } Set & $p=1$ & $p=2$ & $p=3$ & $p=4$ & $p=1$ & $p=2$ & $p=3$ & $p=4$ \\
\hline \hline 1 & -2.4 & -1.6 & 0.0 & 0.3 & 0.6 & 1.8 & 0.0 & -2.7 \\
\hline 2 & 1.1 & 1.6 & 0.0 & -1.7 & 2.2 & 1.2 & 0.0 & -4.7 \\
\hline 3 & 1.3 & -1.0 & 0.0 & -1.8 & 0.0 & -0.6 & 0.0 & 0.9 \\
\hline 4 & 1.2 & 1.2 & 0.0 & 3.7 & -1.7 & -1.3 & 0.0 & 2.5 \\
\hline 5 & -1.0 & -4.1 & 0.0 & -1.8 & -32.1 & -14.9 & 0.0 & -0.2 \\
\hline 6 & 2.3 & 1.0 & 0.0 & -2.6 & 2.0 & 1.6 & 0.0 & -0.4 \\
\hline 7 & -1.4 & 1.3 & 0.0 & -3.1 & -7.6 & -2.7 & 0.0 & 0.4 \\
\hline 8 & -3.7 & -2.2 & 0.0 & -4.0 & -5.2 & 2.1 & 0.0 & 1.6 \\
\hline \hline Overall & -0.3 & -0.5 & 0.0 & -1.4 & -5.2 & -1.6 & 0.0 & -0.3 \\
\hline
\end{tabular}


Table 6 Percentage performance improvement $(+) /$ degradation(-) observed when running FSA(CO) with different values of $q$. Performance with $q=3$ is used as the reference.

\begin{tabular}{|c||c|c|c|c||c|c|c|c|}
\hline \multirow{2}{*}{\multicolumn{1}{|c||}{ Problem }} & \multicolumn{3}{c||}{ Best Performance } & \multicolumn{3}{|c|}{ Average Performance } \\
\cline { 2 - 9 } Set & $q=1$ & $q=2$ & $q=3$ & $q=4$ & $q=1$ & $q=2$ & $q=3$ & $q=4$ \\
\hline \hline 1 & 0.7 & 0.5 & 0.0 & 1.0 & -0.8 & 0.8 & 0.0 & -1.6 \\
\hline 2 & -1.7 & -1.2 & 0.0 & -2.6 & 0.4 & 0.8 & 0.0 & -0.5 \\
\hline 3 & 2.7 & 3.9 & 0.0 & 2.3 & 2.4 & 1.1 & 0.0 & 1.1 \\
\hline 4 & -3.6 & -3.7 & 0.0 & -8.4 & -0.2 & -0.4 & 0.0 & -3.5 \\
\hline 5 & 2.7 & 0.2 & 0.0 & 1.3 & -17.3 & -6.9 & 0.0 & 1.3 \\
\hline 6 & -4.7 & 1.2 & 0.0 & 1.5 & -2.4 & 0.9 & 0.0 & 1.8 \\
\hline 7 & 1.6 & 1.7 & 0.0 & 0.5 & -2.8 & 0.1 & 0.0 & 0.8 \\
\hline 8 & -1.0 & 1.5 & 0.0 & 1.8 & -5.2 & -1.8 & 0.0 & 1.6 \\
\hline \hline Overall & -0.4 & 0.5 & 0.0 & -0.3 & -3.2 & -0.7 & 0.0 & 0.1 \\
\hline
\end{tabular}

